\title{
Palinologia de espécies de Asteraceae de utilidade medicinal para a região dos Campos Gerais, Ponta Grossa, PR, Brasil
}

\author{
Carin Stanski ${ }^{1}$, Melissa Koch Fernandes de Souza Nogueira ${ }^{1}$ e Cynthia Fernandes Pinto da Luz ${ }^{2,3}$
}

Recebido: 22.03.2016; aceito: 7.06.2016

\begin{abstract}
Pollen morphology of species of Asteraceae with medicinal utility from the region of Campos Gerais, Ponta Grossa, Paraná State, Brazil). We analyzed the pollen morphology of ten medicinal species of Asteraceae (Achillea millefolium L., Baccharis trimera (Less.) DC., Bidens pilosa L., Chromolaena laevigata (Lam.) R.M.King \& H.Rob., Emilia sonchifolia (L.) DC., Galinsoga parviflora Cav., Matricaria chamomilla L., Senecio brasiliensis Less (Spreng.), Solidago chilensis Meyen., and Sonchus oleraceus L.) occurring in that region. The pollen grains showed common morphological characteristics such as the presence of 3(4)-colpori and lalongate endoapertures, differing in dimensions of the structures, exine structure, and aspect of the spines. The Asteraceae showed eurypalynous pattern in the region of Campos Gerais and the formed groups can be used in research requiring botanical certification by Palynology in medicinal products derived from flowers of this family.
\end{abstract}

Keywords: Asteraceae, medicinal plants, palynotaxonomy, pollen grains

RESUMO - (Palinologia de espécies de Asteraceae de utilidade medicinal para a região dos Campos Gerais, Ponta Grossa, PR, Brasil). No presente trabalho foi analisada a morfologia polínica de dez espécies medicinais da família Asteraceae (Achillea millefolium L., Baccharis trimera (Less.) DC., Bidens pilosa L., Chromolaena laevigata (Lam.) R.M.King \& H.Rob., Emilia sonchifolia (L.) DC., Galinsoga parviflora Cav., Matricaria chamomilla L., Senecio brasiliensis Less (Spreng.), Solidago chilensis Meyen. e Sonchus oleraceus L.) ocorrentes na referida região. Os grãos de pólen apresentaram características morfológicas comuns como presença de 3(4)-cólporos, endoaberturas lalongadas, diferindo nas dimensões das estruturas, estrutura da exina e no aspecto dos espinhos. A família Asteraceae se mostrou euripolínica na região dos Campos Gerais e os agrupamentos formados podem ser utilizados nas pesquisas que necessitem da certificação botânica pela Palinologia de produtos medicinais oriundos de flores dessa família.

Palavras-chave: Asteraceae, grãos de pólen, plantas medicinais, palinotaxonomia

\section{Introdução}

Segundo o Ministério da Saúde (Brasil, 2006), a Organização Mundial de Saúde (OMS) reconhece que grande parte da população dos países em desenvolvimento, como o Brasil, depende da medicina tradicional, tendo em vista que $80 \%$ desta população utilizam práticas alternativas nos seus cuidados básicos de saúde e $85 \%$ destes utilizam plantas medicinais ou preparações das mesmas. Segundo a OMS, a medicina tradicional engloba o conhecimento técnico e os procedimentos baseados nas teorias, crenças e experiências indígenas de diferentes culturas, sejam ou não explicáveis pela ciência, usados para a manutenção da saúde, como também para a prevenção, diagnose e tratamento de doenças físicas e mentais. Em alguns países utilizam-se indistintamente os termos "medicina complementar", "medicina alternativa", "medicina não convencional" ou "medicina tradicional", em contraponto a "medicina moderna", "medicina científica" ou "medicina convencional" (OMS 2000).

A análise palinológica de produtos medicinais alternativos que contenham partes florais e que estejam disponíveis no comércio formal ou informal se constitui em mecanismo eficaz para subsidiar a

1. Universidade Estadual de Ponta Grossa, Departamento de Biologia Geral, Laboratório de Palinologia, Av. General Carlos Cavalcanti, 4.748, 84030-900 Ponta Grossa, Paraná, Brasil

2. Instituto de Botânica de São Paulo, Núcleo de Pesquisa em Palinologia, Av. Miguel Estéfano, 3.687, Água Funda, 04301-902 São Paulo, São Paulo, Brasil

3. Autor para correspondência: cyluz@yahoo.com.br 
certificação de sua origem botânica, pois evidencia a presença de conjuntos polínicos característicos de determinadas plantas. Para tanto, são necessários catálogos polínicos com fotos e descrições dos grãos de pólen que auxiliem em suas identificações.

Apesar de a família Asteraceae apresentar vários estudos quanto à morfologia polínica são poucos os trabalhos que tratam dos grãos de pólen de plantas medicinais.

A família Asteraceae compreende o grupo de plantas mais numeroso dentro das Angiospermas, com cerca de 23.000 espécies e 17 tribos (Bremer 1994). No Brasil, a família possui 260 gêneros e cerca de 2000 espécies (Souza \& Lorenzi 2005). Segundo APG III (2009), a família Asteraceae está inserida no clado das Asterídeas na ordem Asterales.

$\mathrm{Na}$ região dos Campos Gerais Asteraceae é a família mais rica em número de espécies e indivíduos de acordo com estudos realizados por Kozera et al. (2008) e Dalazoana (2010), os quais identificaram respectivamente 49 e 91 táxons nesta formação vegetal.

Devido a grande diversidade, muitas das espécies de Asteraceae são utilizadas como plantas medicinais por moradores da região (Staniski et al. 2014). De acordo com Souza \& Lorenzi (2012), destacam-se entre as espécies medicinais a carqueja (Baccharis spp.), camomila (Matricaria chamomilla L.), guaco (Mikania spp.) e mil-folhas (Achillea millefolium L.).

Vários grãos de pólen de espécies medicinais de Asteraceae foram descritos anteriormente por Cancelli et al. $(2005,2007,2008,2010)$ a partir de espécimes coletados no Estado do Rio Grande do Sul, incluindo Baccharis trimera (Less.) DC., Bidens pilosa L., Chromolaena laevigata (Lam.) R.M. King \& H. Rob., Eupatorium laevigatum Lam., Galinsoga parviflora Cav., Senecio brasiliensis (Spreng.) Less. e Solidago chilensis Meyen.

Dentre os trabalhos realizados com plantas medicinais destacam-se o trabalho de Galvão et al. (2009) desenvolvido com grãos de pólen de espécies de Asteraceae de utilidade medicinal para a comunidade da Vila Dois Rios, Ilha Grande, município de Angra dos Reis, Rio de Janeiro, Brasil. Neste, os autores destacaram 12 espécies utilizadas medicinalmente pela comunidade, incluindo Matricaria chamomilla L. (sin. Matricaria recutita L.), Sonchus oleraceus (L.) L., Bidens pilosa L. e Solidago chilensis Meyen. Meo \& Khan (2003) descreveram características polínicas de alguns espécimes do gênero Achillea do Paquistão, incluindo Achillea millefolium.
Os grãos de pólen de Mikania micrantha Kunth também foram estudados por Mendonça \& GonçalvesEsteves (2000) em um trabalho com espécies da tribo Eupatorieae ocorrentes na Restinga de Carapebus, município de Carapebus, Rio de Janeiro. Os grãos de pólen de Emilia sonchifolia (L.) DC. ex DC. foram estudados quanto à morfologia estrutural por Adedeji (2005) em um trabalho desenvolvido na Nigéria com três espécies do gênero Emilia Cass.

Deste modo, o presente trabalho teve por objetivo caracterizar os tipos polínicos das Asteraceae medicinais coletadas na região dos Campos Gerais, município de Ponta Grossa, Paraná, Brasil, fornecendo informações das espécies e seu uso medicinal, auxiliando na identificação taxonômica da medicação à base de ervas, contribuindo nos estudos palinológicos e da área médica.

\section{Material e métodos}

De acordo com Melo et al. (2007), a região dos Campos Gerais situa-se na porção centro-leste do Estado do Paraná, abrangendo porções das mesorregiões Centro Oriental e Sudeste Paranaense. Os Campos Gerais distribuem-se como uma faixa de território curva com convexidade para noroeste, com $11.761,41 \mathrm{~km}^{2}$ de extensão, situada entre as coordenadas $23^{\circ} 45^{\prime}$ e $26^{\circ} 15^{\prime}$ de latitude sul e $49^{\circ} 15^{\prime}$ e $50^{\circ} 45^{\prime}$ de longitude oeste, abrangendo pelo menos parte de 22 municípios do Paraná.

O material botânico utilizado foi obtido de flores e botões florais retirados de exsicatas depositadas no Herbário da Universidade Estadual de Ponta Grossa (HUPG), Paraná, Brasil. Foi realizada a seleção das espécies de Asteraceae mais utilizadas medicinalmente na região dos Campos Gerais a partir de levantamento realizado no Herbário HUPG (Staniski et al. 2014). Para cada espécie retirou-se pólen de pelo menos três botões florais a fim de homogeneizar as variações no tamanho dos grãos de pólen (Salgado-Labouriau 1973).

Material examinado: Achillea millefolium L.: BRASIL. ParanÁ: Jaguariaíva, 2-XI-2003, Benini et al. s.n. (HUPG11002). Baccharis trimera (Less.) DC.: BRASIL. ParanÁ: Tibagi, Parque Estadual do Guartelá, 20-VII-1999, Takeda \& Farago s.n. (HUPG9708). Bidens pilosa L.: BRASIL. Paraná: Ponta Grossa, Rio Pitangui, 9-X-2008, Dalazoana et al. s.n. (HUPG13796). Matricaria chamomilla L.: BRASIL. PARANÁ: Ponta Grossa, Universidade Estadual de Ponta Grossa, Campus Uvaranas, 
31-VIII-2009, Rodrigo Moreira s.n. (HUPG155307). Emilia sonchifolia (L.) DC. ex DC.: BRASIL. PARANÁ: Ponta Grossa, Alagados, 20-II-2010, Moro et al. s.n. (HUPG16440). Chromolaena laevigata (Lam.) R.M. King \& H. Rob.: BRASIL. Paraná: Ponta Grossa, Alagados, Vale do Pitangui, 3-III-2010, Moro et al. s.n. (HUPG16442). Galinsoga parviflora Cav.: BRASIL. PARANÁ: Ponta Grossa, Universidade Estadual de Ponta Grossa, Campus Uvaranas, 14-X-2003, Estreechein et al. s.n. (HUPG11123). Senecio brasiliensis (Spreng.) Less.: BRASIL. Paraná: Tibagi, Parque Estadual do Guartelá, 30-X-2003, Moro et al. s.n. (HUPG121402). Solidago chilensis Meyen.: BRASIL. PARANÁ: Ponta Grossa, Vale do Pitangui, 20-III-2010, Moro et al. s.n. (HUPG16123). Sonchus oleraceus L. (L): BRASIL. PARANÁ: Ponta Grossa, Universidade Estadual de Ponta Grossa, Campus Uvaranas, 27-IX-2007, Moro et al. s.n. (HUPG13889).

O material polínico foi preparado segundo o método da acetólise de Erdtman (1952), com as modificações propostas por Melhem et al. (2003), visando a observação em microscopia de luz. Mensurações foram realizadas usando-se o tambor micrométrico de fio móvel OLYMPUS OSM acoplado à ocular do microscópio OLYMPUS BX 50. Foram tomadas aleatoriamente 25 medidas dos eixos polar (P) e equatorial (E) nos grãos de pólen em vista equatorial, distribuídos em cinco lâminas para cada espécie, visando uma uniformidade da amostra. Posteriormente, foi realizado o tratamento estatístico calculando-se a média aritmética $(\mathrm{x})$, o desvio padrão da média $\left(\mathrm{s}_{\mathrm{x}}\right)$, desvio padrão da amostra (s), o coeficiente de variabilidade (CV), o intervalo de confiança a $95 \%$ e a faixa de variação. As medidas das aberturas, espessura e detalhes da ornamentação da exina foram feitas em 10 grãos de pólen tomados ao acaso e calculadas apenas as médias aritméticas (Salgado-Labouriau 1973).

Para uma melhor definição do aspecto da ornamentação dos grãos de pólen obteve-se a relação largura da base e altura dos espinhos obtendo-se índices que definiram classes que variaram entre as espécies como em Coutinho \& Dinis (2007) para espécies de Asteraceae e Barth et al. (2005) para espécies de Cayaponia (Cucurbitaceae). Deste modo, obtiveram-se índices que definiram classes que variaram em: 1) espinhos compridos - com índices iguais a 1,48 (obtidos para espinhos mais altos do que largos na base), 2) índices entre 1,03 e 1,08 foram obtidos para espinhos ligeiramente mais altos do que largos na base; 3) índices entre 0,84 e 0,98 ligeiramente mais largos na base do que altos e, 4) espinhos curtos - com índices abaixo de 0,64 que caracterizaram espinhos muito mais largos na base do que compridos. Caracterizou-se também o aspecto dos ápices dos espinhos em retos ou encurvados, assim como das columelas infrategiladas e cavidades da base destes, principalmente pela observação em microscopia eletrônica de varredura (MEV), diferindo em número e altura entre as espécies.

A terminologia adotada para as descrições polínicas foi a de Barth \& Melhem (1988) com base nas atualizações apresentadas em Punt et al. (2007) e Punt \& Hoen (2009) para as camadas da exina do pólen de Asteraceae.

Os grãos de pólen acetolisados e preparados para o exame em microscopia óptica foram fotografados digitalmente com o fotomicroscópio LEICA DM 2.500 acoplado a uma câmera de vídeo e microcomputador (PC) utilizando-se o software LAS EZ 1.6.0. do Instituto de Botânica. Visando a análise sob microscopia eletrônica de varredura, os grãos de pólen não acetolisados foram espalhados sob a superfície de pedaços de fita dupla-face de carbono que recobriram suportes de alumínio ("stubs"). As amostras foram transferidas para uma bomba á vácuo e metalizadas com uma fina camada de ouro paládio (ca. 150 ângstrons de espessura), sendo posteriormente analisadas em aparelho Shimadzu modelo SSX-550 pertencente ao Laboratório de Microscopia Eletrônica de Varredura do Departamento de Engenharia de Materiais da Universidade Estadual de Ponta Grossa.

\section{Resultados e Discussão}

Seguem as descrições da morfologia polínica das dez espécies de plantas medicinais analisadas, pertencentes aos dez gêneros ocorrentes na região dos Campos Gerais, Paraná, Brasil:

\section{Achillea millefolium L. (Anthemideae)}

Figuras 1-3

Nome vernáculo: mil-folhas. Uso medicinal: infusão preparada com as inflorescências. Utilizada contra infecção das vias respiratórias, febres, problemas estomacais, dores reumáticas e cólicas menstruais (Viganó et al. 2007).

Grãos de pólen de tamanho médio (figura 1, tabelas 1 e 2), isopolares, suboblato (P/E igual a 0,79 ), 3-colporados, âmbito subtriangular, simetria radial, superfície espinhosa (figura 3). 

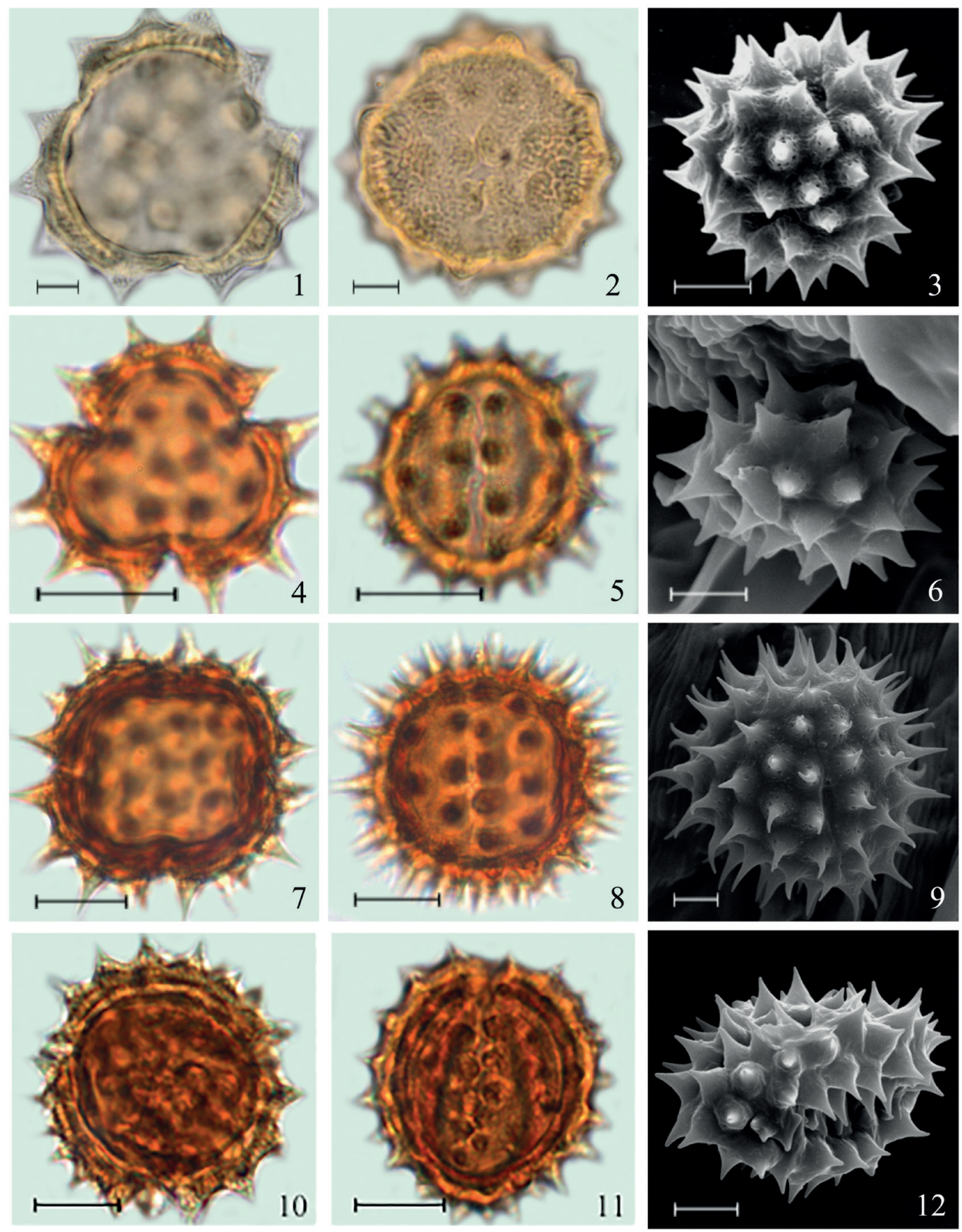

Figuras 1-12. Fotomicrografias e eletromicrografias dos grãos de pólen de espécies medicinais de Asteraceae. Achillea millefolium L. 1. Vista polar: corte óptico. 2. Vista equatorial: abertura. 3. Aspecto geral do grão de pólen (MEV). Baccharis trimera (Less.) DC. 4. Vista polar: corte óptico. 5. Vista equatorial. 6. Aspecto geral do grão de pólen (MEV). Bidens pilosa L. 7. Vista polar: Corte óptico. 8. Vista equatorial. 9. Aspecto geral do grão de pólen (MEV). Chromolaena laevigata (Lam.) R.M. King \& H. Rob. 10. Vista polar: Corte óptico. 11. Vista equatorial: corte óptico. 12. Aspecto geral do grão de pólen (MEV). Figuras 1-3, 6, 9, escala = 5 $\mu$ m; Figuras 4-5, 7-8 , 10-12, escala $=10 \mu \mathrm{m}$.

Figures 1-12. Photomicrographs and electron micrographs of pollen grains of medicinal species of Asteraceae. Achillea millefolium L. 1. Polar view: optical section. 2. Equatorial view: aperture. 3. General aspect of the pollen grain (SEM). Baccharis trimera (Less.) DC. 4. Polar view: optical section. 5. Equatorial view . 6. General aspect of the pollen grain (SEM). Bidens pilosa L. 7. Polar view: optical section. 8. Equatorial view . 9. General aspect of the pollen grain (SEM). Chromolaena laevigata (Lam.) R.M. King \& H. Rob.. 10. Polar view: optical section . 11. Equatorial view: optical section. 12. General aspect of the pollen grain (SEM). Figures $1-3,6,9$, scale $=5 \mu$ m; Figures 4-5, 7-8, 10-12, scale $=10 \mu \mathrm{m}$. 
Aberturas: cólporos longos e muito estreitos (tabela 2), com extremidades de difícil visualização e sem constrição mediana. Endoabertura lalongada (tabela 2), com constrição mediana (figura 2).

Exina: sexina separada da nexina por um nítido espaço vazio (cávea); espinhos isolados, muito mais largos na base do que altos (índice 0,61) (tabela 3), com ápice agudo, com cerca de três pequenas cavidades na base, columelas infrategiladas mais altas na base dos espinhos. A espessura da sexina 1 e 2 é o triplo da nexina (tabela 3 ).

\section{Baccharis trimera (Less.) DC. (Astereae)}

Figuras 4-6

Nome vernáculo: carqueja. Uso medicinal: infusão de folhas picadas. Recomendada para afecções estomacais, intestinais e hepáticas, garganta inflamada e contra vermes intestinais (Viganó et al. 2007, Vendruscolo et al. 2005).

Grãos de pólen de tamanho pequeno (figura 4, tabelas 1 e 2), isopolares, prolato esferoidal (P/E igual a 1,02), 3-colporados, âmbito subcircular, simetria radial, superfície espinhosa (figura 6).

Aberturas: cólporos longos e largos, com extremidades afiladas e sem constrição mediana. Endoabertura muito lalongada (tabela 2), com constrição mediana (figura 5).

Exina: sexina separada da nexina por um espaço vazio (cávea) de difícil visualização; espinhos isolados e compridos, ligeiramente mais altos do que largos (índice 1,03), com ápice agudo, com três pequenas cavidades na base, columelas infrategiladas mais altas na base dos espinhos. A espessura da sexina 1 e 2 é muito maior do que da nexina (tabela 3 ).

\section{Bidens pilosa L. (Heliantheae)}

Figuras 7-9

Nome vernáculo: picão-preto. Uso medicinal: infusão preparada com a planta inteira. Utilizada contra febres, problemas do fígado, infecções urinárias e resfriados, como antibiótico e anti-inflamatório (Vendruscolo et al. 2005, Galvão et al. 2009, Staniski 2011).

Grãos de pólen de tamanho pequeno (figura 7, tabelas $1 \mathrm{e} 2$ ), isopolares, prolato esferoidal ( $\mathrm{P} / \mathrm{E}$ igual a 1,01), (3)4-colporados, 90\% de grãos 4-colporados (figura 7), âmbito subtriangular ou quadrangular, simetria radial, superfície espinhosa (figura 9).

Aberturas: cólporos longos e muito estreitos com extremidades afiladas e sem constrição mediana. Endoabertura lalongada (tabela 2) de difícil visualização, com constrição mediana $(50 \%)$ e sem constrição mediana (50\%), apresentando extremidades afiladas assimétricas (figura 8).

Exina: sexina separada da nexina por um nítido espaço vazio (cávea); espinhos isolados, compridos, ligeiramente mais largos na base do que altos (índice 0,97), com ápice agudo, com mais de três pequenas cavidades na base, columelas infrategiladas mais altas na base dos espinhos. A espessura da sexina 1 e 2 é igual a da nexina (tabela 3).

4. Chromolaena laevigata (Lam.) R.M.King \& H.Rob. (Eupatorieae)

Figuras 10-12

Tabela 1. Caracterização morfológica geral dos grãos de pólen das espécies medicinais de Asteraceae ocorrentes em Campos Gerais, Ponta Grossa, PR, Brasil.

Table 1. General morphological characterization of pollen grains of the medicinal species of Asteraceae occurring in the region of Campos Gerais, Paraná State, Brazil.

\begin{tabular}{lcccc}
\hline Táxons & Tamanho & Abertura & Cólporo & Endoabertura \\
\hline Achillea millefolium & médio & 3-colporados & longos e muito estreitos & lalongada \\
Baccharis trimera & pequeno & 3-colporados & longos e largos & muito lalongada \\
Bidens pilosa & pequeno & (3)4-colporados & longos e muito estreitos & lalongada \\
Chromolaena laevigata & pequeno & 3(4)-colporados & longos e estreitos & circular \\
Emilia sonchifolia & médio & 3-colporados & longos e muito estreitos & circular \\
Galinsoga parviflora & pequeno & 3(4)-colporados & longos e muito estreitos & lalongada \\
Matricaria chamomilla & pequeno & 3-colporados & longos e muito estreitos & lalongada \\
Senecio brasiliensis & pequeno & 3-colporados & longos e estreitos & lalongada \\
Solidago chilensis & pequeno & 3-colporados & médios e estreitos & lalongada \\
Sonchus oleraceus & médio & 3-4-colporados & difícil visualização & lalongada \\
\hline
\end{tabular}




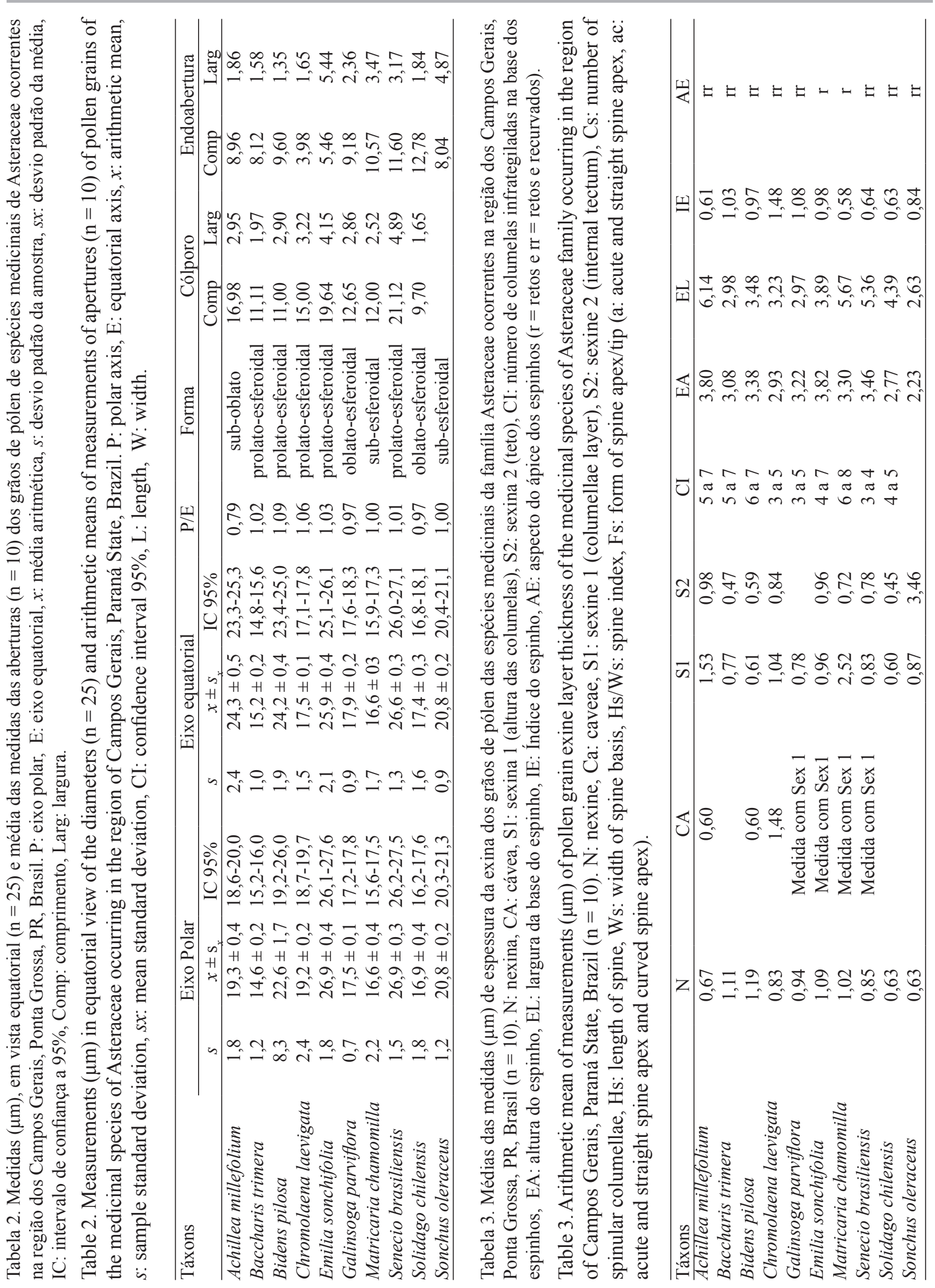


Nome vernáculo: cambará. Uso medicinal: utilizada na forma de infusão. Possui propriedades antibacterianas e antifúngicas (Murakami 2009).

Grãos de pólen de tamanho pequeno (figura 10, tabelas 1 e 2), isopolares, prolato esferoidais ( $\mathrm{P} / \mathrm{E}$ igual a 1,06), 3-(4)-colporados, $80 \%$ de 3 -colporados, âmbito circular, simetria radial, superfície espinhosa (figura 12).

Aberturas: cólporos longos e estreitos (tabela 2) com extremidades afiladas e sem constrição mediana. Endoabertura circular (figura 11).

Exina: sexina separada da nexina por um nítido espaço vazio (cávea); espinhos isolados, ligeiramente mais largos na base do que altos (índice 0,90) (tabela 3), com ápice agudo, com três pequenas cavidades na base, columelas infrategiladas mais altas na base dos espinhos. A espessura da sexina 1 e 2 é mais que o dobro da nexina (tabela 3 ).

\section{Emilia sonchifolia (L.) DC. (Senecioneae)}

Figuras 13-15

Nome vernáculo: serralha. Uso medicinal: utilizada na forma de infusão de folhas e flores. Contra gripes, resfriados, dores no corpo e como emagrecedor (Teixeira \& Melo 2006).

Grãos de pólen de tamanho médio (figura 13, tabelas $1 \mathrm{e} 2$ ), isopolares, prolato esferoidais (P/E igual a 1,03), 3-colporados, âmbito subcircular, simetria radial, superfície espinhosa (figura 15).

Aberturas: cólporos longos e muito estreitos, com extremidades de difícil visualização e sem constrição mediana (figura 14). Endoabertura circular (tabela 2).

Exina: sexina separada da nexina por um espaço vazio (cávea), espinhos isolados, ligeiramente mais largos na base do que altos (índice 0,98) (tabela 3), com ápice agudo, com cerca de três pequenas cavidades na base, columelas infrategiladas mais altas na base dos espinhos. A espessura da sexina 1 e 2 é o dobro da nexina (tabela 3 ).

\section{Galinsoga parviflora Cav. (Heliantheae)}

Figuras 16-18

Nome vernáculo: picão-branco. Uso medicinal: infusão das folhas. Utilizada contra doenças bronco pulmonares e do aparelho digestivo e, também é cicatrizante (Vendruscolo et al. 2005).

Grãos de pólen de tamanho pequeno (figura 16, tabelas 1 e 2), isopolares, oblato esferoidais (P/E igual a 0,97), 3-(4)-colporados, $80 \%$ de grãos 3-colporados, âmbito subtriangular ou quadrangular, simetria radial, superfície espinhosa (figura 18).

Aberturas: cólporos longos e muito estreitos (tabela 2) com extremidades afiladas e sem constrição mediana (figura 17). Endoabertura lalongada de difícil visualização, com constrição mediana (50\%) ou sem constrição mediana (50\%).

Exina: sexina separada da nexina por um espaço vazio (cávea) de difícil visualização; espinhos isolados, compridos, ligeiramente mais largos na base do que altos (índice 1,08) (tabela 3), com ápice agudo, com mais de três pequenas cavidades na base, columelas infrategiladas mais altas na base dos espinhos. A espessura da sexina 1 é igual a da nexina (tabela 3).

7. Matricaria chamomilla L. (Anthemideae)

Figuras 19-21

Nome vernáculo: camomila. Uso medicinal: infusão de flores. Utilizada contra cólicas de crianças por ser digestiva e calmante (Viganó et al. 2007, Galvão et al. 2009, Staniski 2011).

Grãos de pólen de tamanho pequeno (figura 19, tabelas 1 e 2), isopolares, subesferoidal (P/E igual a 1,00), 3-colporados, âmbito circular, simetria radial, superfície espinhosa (figura 21).

Aberturas: cólporos longos e muito estreitos com extremidades afiladas e sem constrição mediana (figura 20). Endoabertura lalongada (tabela 2), sem constrição mediana.

Exina: sexina separada da nexina por um espaço vazio (cávea) de difícil visualização, espinhos isolados, muito mais largos na base do que altos (índice 0,58) (tabela 3), com ápice agudo, com cerca de três pequenas cavidades na base, columelas infrategiladas mais altas na base dos espinhos. A espessura da sexina 1 e 2 é o triplo da nexina (tabela 3 ).

\section{Senecio brasiliensis Less (Spreng.) (Senecioneae)} Figuras 22-24

Nome vernáculo: maria-mole, micuim. Uso medicinal: folhas secas que são utilizadas na cura de feridas, queimaduras e alergias (Vendruscolo et al. 2005).

Grãos de pólen de tamanho médio (figura 22, tabelas 1 e 2), isopolares, prolato-esferoidais $(\mathrm{P} / \mathrm{E}$ igual a 1,01), 3-colporados, âmbito subcircular, simetria radial, superfície espinhosa (figura 24). 
Aberturas: cólporos longos e largos (tabela 2) com extremidades afiladas e sem constrição mediana (figura 23). Endoabertura lalongada de difícil visualização, com constrição mediana (50\%) ou sem constrição mediana $(50 \%)$.
Exina: sexina separada da nexina por um espaço vazio (cávea) de difícil visualização; espinhos isolados, muito mais largos na base do que altos (índice 0,64) (tabela 3), com ápice agudo, com cerca de 4 pequenas cavidades na base, columelas infrategiladas mais altas
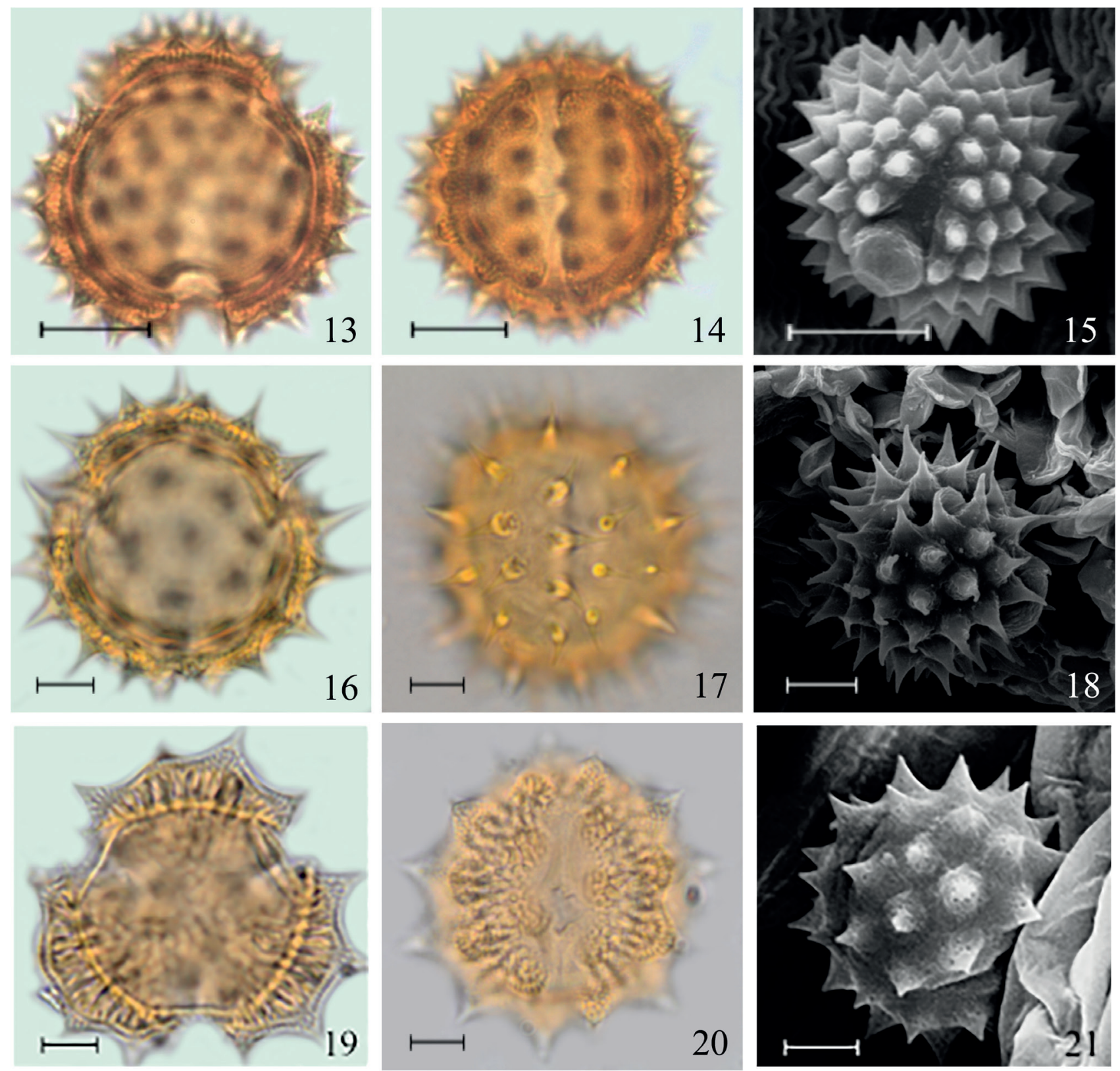

Figuras 13-21. Fotomicrografias e eletromicrografias dos grãos de pólen de espécies medicinais de Asteraceae. Emilia sonchifolia (L.) DC. 13. Vista polar: corte óptico. 14. Vista equatorial: abertura. 15. Aspecto geral do grão de pólen (MEV). Galinsoga parviflora Cav. 16. Vista polar: corte óptico. 17. Vista equatorial. 18. Aspecto geral do grão de pólen (MEV). Matricaria chamomilla L. 19. Vista polar: corte óptico. 20. Vista equatorial: corte óptico. 21. Aspecto geral do grão de pólen (MEV). Figuras 16-21, escala = 5 $\mu$ m; Figuras 13-15, escala $=10 \mu \mathrm{m}$.

Figures 13-21. Photomicrographs and electron micrographs of pollen grains of medicinal species of Asteraceae. Emilia sonchifolia (L.) DC. 13. Polar view: optical section. 14. Equatorial view: aperture. 15. General aspect of the pollen grain (SEM). Galinsoga parviflora Cav. 16. Polar view: optical section. 17. Equatorial view. 18. General aspect of the pollen grain (SEM). Matricaria chamomilla L. 19. Polar view: optical section. 20. Equatorial view: optical section. 21. General aspect of the pollen grain (SEM). Figures 16-21, scale = $5 \mu \mathrm{m}$; Figures 13-15, scale $=10 \mu \mathrm{m}$. 
na base dos espinhos. A espessura da sexina 1 é o dobro da nexina (tabela 3 ).

\section{Solidago chilensis Meyen. (Astereae)}

Figuras 25-27

Nome vernáculo: arnica. Uso medicinal: infusão da planta inteira. É usada como adstringente, cicatrizante, em torções e inflamações (Schardong \& Cervi 2000, Galvão et al. 2009).

Grãos de pólen de tamanho pequeno (figura 25, tabelas 1 e 2), isopolares, oblato esferoidais (P/E igual a 0,97), 3-colporados, âmbito subcircular, simetria radial, superfície espinhosa (figura 27).

Aberturas: cólporos médios e estreitos (tabela 2) com extremidades afiladas e sem constrição mediana (figura 26). Endoabertura lalongada (tabela 2), sem constrição mediana.

Exina: sexina separada da nexina por um nítido espaço vazio (cávea); espinhos isolados, curtos, muito mais largos na base do que altos (índice 0,63) (tabela 3), com ápice agudo, com três pequenas cavidades na base, columelas infrategiladas mais altas na base dos espinhos. A espessura da sexina 1 e 2 é mais que o dobro da nexina (tabela 3 ).

\section{Sonchus oleraceus (L.) L. (Lactuceae)}

Figuras 28-30

Nome vernáculo: serralha. Uso medicinal: utilizada na forma de infusão. Indicada para problemas estomacais, hepáticos e intestinais (Vendruscolo \& Mentz 2006, Galvão et al. 2009).

Grãos de pólen de tamanho médio (figura 28, tabelas 1 e 2), isopolares, subesferoidais ( $\mathrm{P} / \mathrm{E}$ igual a 1,00), 3-4-colporados, âmbito subtriangular ou quadrangular, simetria radial, superfície equinolofada, sem lacuna polar (figura 30).

Aberturas: cólporos estreitos de difícil visualização (figura 29). Endoabertura lalongada (tabela 2), sem constrição mediana.

Exina: exina equinolofada, com muros altos e largos, com presença de muitas perfurações e, sobre os muros presença de espinhos isolados, ligeiramente mais largos na base do que altos (índice 0,84) (tabela 3). Sexina entre espinhos é mais que o dobro da nexina (tabela 3).

Através dos resultados obtidos observou-se que a família Asteraceae dos Campos Gerais possui uma variedade significativa de tipos polínicos. Dentre os grãos de pólen das espécies medicinais estudadas, as características observadas foram o tamanho de pequeno a médio, a forma sub-oblata, prolatoesferoidal, sub-esferoidal ou oblato-esferoidal; 3(4) cólporos e endoaberturas lalongadas ou circulares. A ornamentação das espécies estudadas é espinhosa (na maioria das espécies) e com o padrão equinolofado apenas em Sonchus oleraceus.

Entre os estudos palinológicos de Asteraceae, Achillea millefolium foi caracterizada por Meo \& Khan (2003) com grãos de pólen de tamanho médio, isopolares, com aberturas elípticas ou circulares, com sexina muito mais espessa que a nexina. Confrontando com os dados aqui obtidos, os grãos de pólen analisados possuem semelhanças quanto ao tamanho, simetria e espessura da exina, diferindo no tipo de abertura o qual foi descrito aqui como cólporos longos e muito estreitos e com endoabertura lalongada com constrição mediana.

Baccharis trimera está inserida no tipo polínico Baccharis defenido por Stix (1960) e, em Cancelli et al. (2005), os grãos de pólen dessa espécie do Rio Grande do Sul foram descritos como pequenos, prolato-esferoidais, com endoabertura lalongada e cólporos bem visíveis. Melhem et al. (2003) descreveram a espécie coletada em Campos do Jordão (SP) possuindo grãos de pólen com âmbito subcircular, divergindo dos autores citados. Nossos resultados corroboraram os dos últimos autores.

Osgrãos de pólen de Bidens pilosa caracterizaram-se como 4-colporados, corroborando os resultados obtidos por Ikuse (1956), Melhem et al. (1979), Sanchez \& Lupo (2009) e Galvão et al. (2009). Contudo, Huang (1972) e Cancelli et al. (2007, 2010) descreveram que a espécie possui predominância de grãos de pólen 3-colporados, no entanto, verificaram a existência de variabilidade morfopolínica entre diferentes espécimes. Na presente pesquisa, apesar dos grãos de pólen de Bidens pilosa possuírem dimensões menores e apenas 4-aberturas, se enquadraram no tipo polínico Hetianthus de Stix (1960) ou Compositae M (Montanoa) de Barth (1989).

Os grãos de pólen de Matricaria chamomilla (sin. Camomilla recutita) foram descritos por Wodehouse (1935), sendo os resultados semelhantes aos obtidos por Galvão et al. (2009), com grãos de pólen oblatoesferoidais. Galvão et al. (2009) descreveram os grãos de pólen com tamanho médio, oblatoesferoidais, 3-colporados e com superfície espinhosa. Já os grãos de pólen aqui analisados foram pequenos, subesferoidais, com colpos longos e muito estreitos. 
Sonchus oleraceus foi descrita por Wodehouse (1935) com grãos de pólen oblato-esferoidais, com maior ocorrência de 4-colporados, mas apresentando também 3 cólporos. Perveen (1999) descreveu-os como suboblatos e com forma octogonal em vista polar. Díez et al. (1999) os descreveram como equinolofados, de tamanho pequeno à médio e 3(4) colporados. Galvão et al. (2009) caracterizou-os
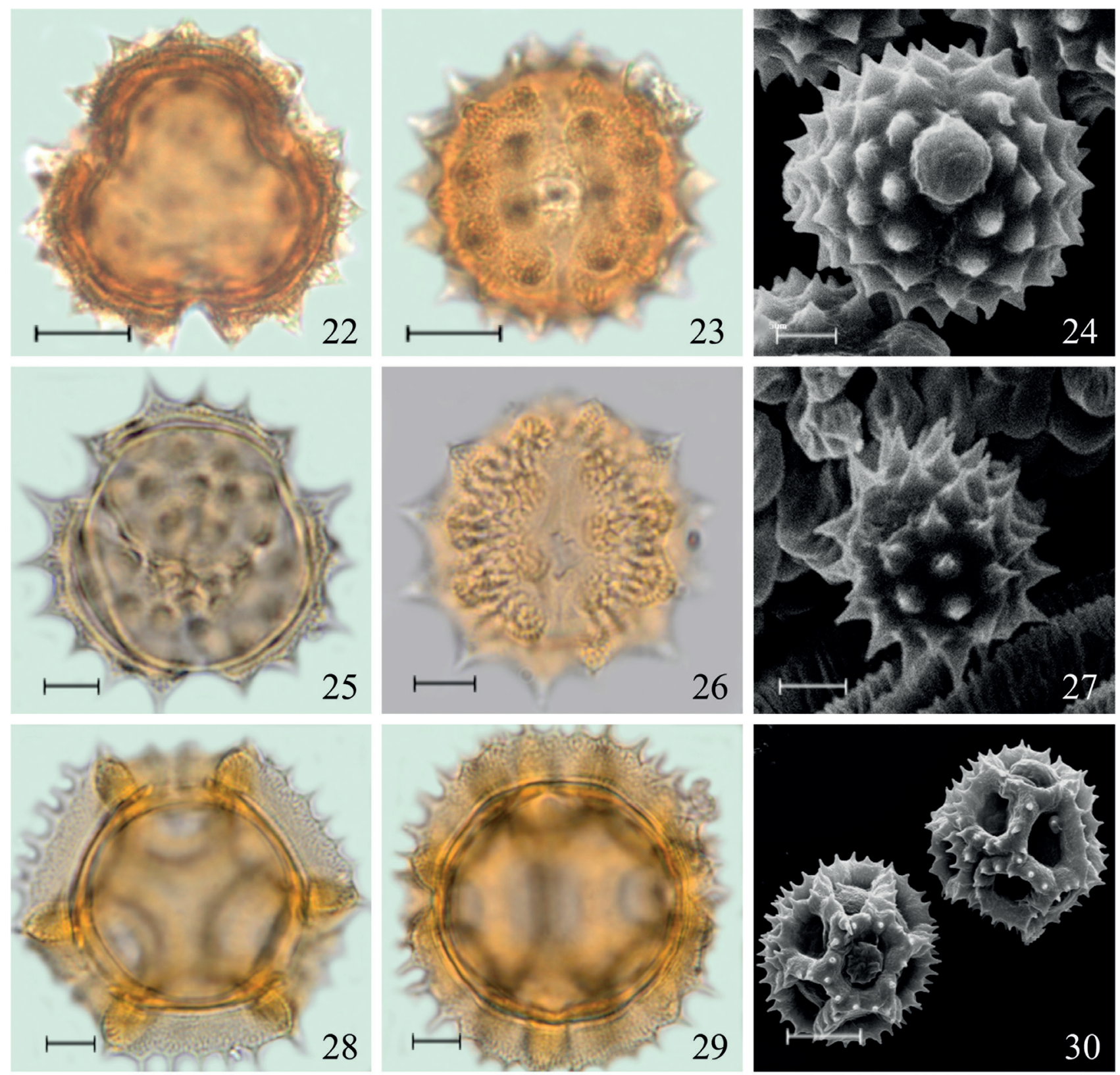

Figuras 22-30. Fotomicrografias e eletromicrografias dos grãos de pólen de espécies medicinais de Asteraceae. Senecio brasiliensis Less (Spreng.). 22. Vista polar: corte óptico. 23. Vista equatorial: abertura. 24. Aspecto geral do grão de pólen (MEV). Solidago chilensis Meyen 25. Vista polar: Corte óptico. 26. Vista equatorial: corte óptico. 27. Aspecto geral do grão de pólen (MEV). Sonchus oleraceus L. 28. Vista polar: corte óptico. 29. Vista equatorial. 30. Aspecto geral dos grãos de pólen (MEV). Figuras 24-29, escala = $5 \mu$ m; Figuras $22-23,30$, escala $=10 \mu \mathrm{m}$.

Figures 22-30. Photomicrographs and electron micrographs of pollen grains of medicinal species of Asteraceae. Senecio brasiliensis Less (Spreng.). 22. Polar view: optical section. 23. Equatorial view: aperture. 24. General aspect of the pollen grain (SEM). Solidago chilensis Meyen . 25. Polar view: optical section. 26. Equatorial view: optical section. 27. General aspect of the pollen grain (SEM). Sonchus oleraceus L. 28. Polar view: optical section. 29. Equatorial view. 30. General aspect of the pollen grains (SEM). Figures 24-29, scale = $5 \mu \mathrm{m}$; Figures 22-23, 30, scale $=10 \mu \mathrm{m}$. 
como grãos de pólen médios, oblato-esferoidais, com superfície equinolofada e com 3(4) poros, aberturas essas que não coincidem com a maioria das descrições para a espécie. As descrições polínicas apresentadas pelos outros autores citados são semelhantes às apresentadas aqui, diferindo apenas na forma ou no âmbito. No entanto, os grãos de pólen apresentaram tamanho pequeno e cólporos, ao contrário do tamanho médio e das aberturas poradas descritas em Galvão et al. (2009).

Os grãos de pólen de Emilia sonchifolia e Senecio brasiliensis da tribo Senecioneae, apesar de pertencerem a gêneros diferentes, possuem similaridades quanto ao tamanho, forma e âmbito (médio, prolato-esferoidal e subcircular). Emilia sonchifolia foi descrita por Adedeji (2005) com grãos de pólen 3-colporados, e foi a única espécie do gênero que apresentou grãos de polén 4-colporados e tamanho médio. Aqui se observaram apenas grãos de pólen 3-colporados. Já em Cancelli et al. (2010), S. brasiliensis foi descrita apresentando pólen de tamanho médio e forma prolato-esferoidal, corroborando os dados aqui descritos.

Solidago chilensis analisado em trabalhos de Cancelli et al. $(2005,2010)$ apresentaram grãos de pólen médios, oblato-esferoidais e 3-colporados, diferindo no tamanho e âmbito descritos aqui como pequeno e subcircular, respectivamente.

Galinsoga parviflora foi analisada por Cancelli et al. (2010) com grãos de pólen médios, prolato-esferoidais e 3-colporados, divergindo do presente estudo, pois aqui foram pequenos, oblatoesferoidais e com 3 a 4-cólporos. O mesmo autor ainda descreveu os grãos de pólen de Chromolaena laevigata (sin. Eupatorium laevigatum) como de tamanho médio, os quais foram descritos aqui como pequenos.

Através dos resultados obtidos pode-se concluir que as espécies medicinais estudadas apresentaram ampla variedade morfopolínica, cujos grãos de pólen abrangeram características bem definidas entre as espécies. A Palinologia foi considerada, portanto, importante ferramenta na identificação das espécies para estudos taxonômicos, cujos resultados podem servir também na certificação da origem botânica dos medicamentos a base de ervas que utilizem as partes florais dessas espécies. Deste modo, confirmaram-se os dados apresentados em Erdtman (1952) que tratou Asteraceae como euripolínica devido às variações características dentro da família.

\section{Agradecimentos}

Ao Laboratório de Botânica, de Palinologia e de Microscopia Eletrônica de Varredura da Universidade Estadual de Ponta Grossa (UEPG), Paraná, pelas eletromicrografias. Ao Instituto de Botânica, Secretaria do Meio Ambiente do Estado de São Paulo, pelo uso das instalações e equipamentos do Núcleo de Pesquisa em Palinologia. Ao Dr. José Ivanildo de Souza do Núcleo de Pesquisa em Micologia do Instituto de Botânica pelo uso do microscópio LEICA DM 2500. À Fundação Araucária, pelo apoio financeiro através da concessão da bolsa BEC-PROEX-UEPG concedida à primeira Autora. Agradecemos ao Conselho Nacional de Desenvolvimento Científico e Tecnológico (CNPq) pela bolsa de produtividade em pesquisa concedida à terceira autora (Processo número 301134/2013-8).

\section{Literatura citada}

Adedeji, O. 2005. Pollen morphology of the three species of the genus Emilia CASS. (Asteraceae) from Nigeria. Journal of Botany 15: 1-9.

Angiosperm Phylogeny Group. 2009. An update of the phylogeny group classification for the orders and families of flowering plants: APG III. Botanical Journal of the Linnean Society 161: 105-21.

Barth, O.M. 1989. O pólen no mel brasileiro. Gráfica Luxor, Rio de Janeiro.

Barth, O.M. \& Melhem, T.S. 1988. Glossário ilustrado de palinologia. Universidade Estadual de Campinas, Campinas.

Barth, O.M., Luz, C.F.P. \& Gomes-Klein, V.L. 2005. Pollen morphology of Brazilian species of Cayaponia Silva Manso (Cucurbitaceae, Cucurbiteae). Grana 44: 129-136.

Bremer, K. 1994. Asteraceae: Cladistics \& Classification. Timber Press, Portland.

Cancelli, R.R. 2008. Palinologia de Asteraceae: morfologia polínica e suas implicações nos registros do Quaternário do Rio Grande do Sul. Dissertação de Mestrado, Universidade Federal do Rio Grande do Sul, Porto Alegre, Rio Grande do Sul.

Cancelli, R.R., Evadt, A.C.P. \& Bauermann, S.G. 2007. Contribuição à morfologia polínica da família Asteraceae Martinov. no Rio Grande do Sul - Parte I. Pesquisas, Série Botânica 58: 347-374.

Cancelli, R.R., Macedo, R.B, Guerreiro, C.T. \& Bauermann, S.G. 2005. Diversidade Polínica em Asteraceae Martinov da Fazenda São Maximiniano, Guaíba, RS. Pesquisas, Série Botânica 56: 209-228.

Cancelli, R.R., Evaldt, A.C.P., Bauermann, S.G., Souza, P.A., Bordignon, S.A.L. \& Matzenbacher, N.I. 2010. Catálogo palinológico de táxons da família Asteraceae Martinov, no Rio Grande do Sul, Brasil. Iheringia, Série Botânica 65: 201-280. 
Coutinho, A. P. \&. Dinis, A. M. 2007. A contribution to the ultrastructural knowledge of the pollen exine in subtribe Inulinae (Inuleae, Asteraceae). Plant Systematics and Evolution 269: 159-170.

Dalazoana, K. 2010. Especialização dos campos nativos na escarpa devoniana do Parque Nacional dos Campos Gerais, Ponta Grossa, Paraná, Brasil. Dissertação de mestrado, Universidade Estadual de Ponta Grossa, Ponta Grossa, Paraná.

Díez, M.J., Mejías, J. A. \& E. Moreno-Socías. 1999. Pollen morphology of Sonchus and related genera, and a general discussion. Plant Systematics and Evolution 214: 91-102.

Erdtman, G. 1952. Pollen morphology and plant taxonomyAngiosperms. Almqvist \& Wiksell, Stockholm.

Galvão, M.N., Pereira, A.C.M., Gonçalves-Esteves, V. \& Esteves, R.L. 2009. Palinologia de espécies de Asteraceae de utilidade medicinal para a comunidade da Vila Dois Rios, Ilha Grande, Angra dos Reis, RJ, Brasil. Acta botanica brasilica 23: 247-258.

Huang, T.C. 1972. Pollen fl ora of Taiwan. National Taiwan University, Taiwan.

Ikuse, M. 1956. Pollen grains of Japan. Hirokawa Publishing Co, Tokyo.

Kozera, C., Kuuniyoshi, Y.S., Galvão, F. \& Curcio, G.R. 2008. Composição florística de uma formação pioneira com influência fluvial em Balsa Nova, PR, Brasil. Floresta 39: 309-322.

Melhem, T.S., Silvestre, M.S.F. \& Makino H. 1979. Grãos de pólen de plantas alergógenas: Compositae. Hoehnea 8: 73-100.

Melhem, T.S., Cruz, M.A.V., Corrêa, A.M.S., MakinoWatanabe, H., Silvestre-Capelato, M.S. \& Esteves, V.L.G. 2003. Variabilidade polínica em plantas de Campos do Jordão (São Paulo, Brasil). Boletim do Instituto de Botânica 16: 1-104.

Melo, M.S., Moro, R.S. \& Guimarães, G.B. 2007. Os Campos Gerais Do Paraná. In: M. Melo, S.R.S. Moro \& G.B. Guimarães (eds.). Patrimônio Natural dos Campos Gerais do Paraná. Editora UEPG, Ponta Grossa, pp.17-22.

Mendonça, C.B. \& Gonçalves-Esteves, V. 2000. Morfologia polínica de algumas espécies da tribo Vernonieae (Compositae Giseke) da Restinga de Carapebus, Rio de Janeiro. Hoehnea 27: 131-142.

Meo, A.A. \& Khan, M.A. 2003b. Pollen morphology of Achillea (Compositae-Anthemideae) species from Pakistan. Pakistan Journal of Weed Sciences Research 9: 253-258.

Ministério da Saúde. 2006. Política Nacional de Práticas Integrativas e Complementares no SUS, PNPIC, SUS, Ministério da Saúde, Brasília.

Murakami, C. 2009. Estudo da composição química e atividades biológicas de óleos voláteis de Chromolaena laevigata (Lam.) King \& Rob. em diferentes fases fenológicas. Dissertação de Mestrado, Instituto de Botânica, São Paulo.

OMS - Organización Mundial de la Salud. 2000. Pautas generales para las metodologías de investigación y evaluación de la medicina tradicional. Ginebra, WHO/ EDM/TRM/2000.
Perveen,A. 1999. Contributions to the Pollen morphology of the family Compositae. Turkish Journal of Biology 23: 523-535.

Punt, W. \& Hoen, P.P. 2009. The Northwest European Pollen Flora, 70: Asteraceae-Asteroideae. Review of Palaeobotany and Palynology 157: 22-183.

Punt, W., Hoen, P.P., Blackmore, S., Nilsson, S. \& Le Thomas, A. 2007. Glossary of Pollen and Spore Terminology. Review of Palaeobotany and Palynology 143: 1-81.

Salgado-Labouriau, M.L. 1973. Contribuição à Palinologia dos Cerrados. Academia Brasileira de Ciências, Rio de Janeiro.

Sanchez, A.C. \& Lupo, L.C. 2009. Asteraceae de interesse em Melissopalinologia. Bosque montano de Las Yungas (Jujuy - Argentina). Boletim da Sociedade Argentina de Botânica 44: 57-64.

Schardong, R.M.F. \& Cervi,A.C. 2000. Estudos etnobotânicos das plantas de uso medicinal e místico na comunidade de São Benedito, Bairro São Francisco, Campo Grande, MS, Brasil. Acta Biológica Paranaense 29: 187-217.

Souza, V.C. \& Lorenzi, H. 2005. Botânica sistemática: guia ilustrado para identificação das famílias de Angiospermas da flora brasileira, baseado em APG II. Ed. Instituto Plantarum, Nova Odessa.

Souza, V.C. \& Lorenzi. H. 2012. Botânica Sistemática: Guia ilustrado para identificação das famílias de Fanerógamas nativas e exóticas no Brasil, baseado em APG III. 3 ed. Instituto Plantarum, Nova Odessa.

Staniski, A. 2011. Os conhecimentos tradicionais no uso de plantas medicinais: reflexões a partir da comunidade faxinal Sete Saltos de Baixo/Ponta Grossa - PR. Monografia de Graduação, Universidade Estadual de Ponta Grossa, Ponta Grossa, Paraná.

Staniski, A., Floriani, N. \& Strachulski, J. 2014. Estudo etnobotânico de plantas medicinais na comunidade faxinalense Sete Saltos de Baixo, Ponta Grossa - PR. Terr@Plural 8: 321-240.

Stix, E. 1960. Pollen morphologische untersuchungen an Compositen. Grana Palynologica 2: 41-104.

Teixeira, S.A. \& Melo, J.I.M. 2006. Plantas medicinais utilizadas no município de Jupi, Pernambuco, Brasil. Iheringia, Série Botânica 61: 5-11.

Wodehouse, R.P. 1935. Pollen Grains: Their structure, identification and significance in science and medicine. McGraw-Hill Book Co., New York.

Vendruscolo, G.S. \& Mentz, L.A. 2006. Levantamento etnobotânico das plantas utilizadas como medicinais por moradores do bairro Ponta Grossa, Porto Alegre, Rio Grande do Sul, Brasil. Theringia, Série Botânica 61: 83-103.

Vendruscolo, G.S., Simões, C.M.O. \& Mentz, L.A. 2005. Etnobotânica no Rio Grande do Sul: análise comparativa entre o conhecimento original e atual sobre as plantas medicinais nativas. Pesquisas, Série Botânica 56: 285-322.

Viganó, J., Viganó, J.A. \& Cruz-Silva, C.T.A. 2007. Utilização de plantas medicinais pela população da região urbana de Três Barras do Paraná. Acta Scientiarum. Health Sciences 29: 51-58. 